\title{
RANK-ligand (RANKL) expression in young breast cancer patients and during pregnancy
}

\author{
Hatem A Azim Jr ${ }^{1,2^{*}}$, Fedro A Peccatori ${ }^{3}$, Sylvain Brohée ${ }^{1}$, Daniel Branstetter ${ }^{4}$, Sherene Loi ${ }^{5}$, Giuseppe Viale ${ }^{6}$, \\ Martine Piccart ${ }^{7}$, William C Dougall ${ }^{8}$, Giancarlo Pruneri ${ }^{6}$ and Christos Sotiriou ${ }^{1,7}$
}

\begin{abstract}
Introduction: RANKL is important in mammary gland development during pregnancy and mediates the initiation and progression of progesterone-induced breast cancer. No clinical data are available on the effect of pregnancy on RANK/RANKL expression in young breast cancer patients.
\end{abstract}

Methods: We used our previously published dataset of 65 pregnant and 130 matched young breast cancer patients with full clinical, pathological, and survival information. $85 \%$ of patients had available transcriptomic data as well. RANK/RANKL expression by immunohistochemistry using $\mathrm{H}$-score on the primary tumor and adjacent normal tissue was performed. We examined the difference in expression of RANK/RANKL between pregnant and non-pregnant patients and their association with clinicopathological features and prognosis. We also evaluated genes and pathways associated with RANK/RANKL expression on primary tumors.

Results: RANKL but not RANK expression was more prevalent in the pregnant group, both on the tumor and adjacent normal tissue, independent of other clinicopathological factors (both $P<0.001$ ). 18.7\% of pregnant and $5.3 \%$ of non-pregnant patients had tumors showing $\geq 10 \%$ of cells with $3+$ RANKL expression. RANKL expression was significantly higher in progesterone receptor-positive, and luminal A-like tumors, with negative correlation with Ki-67 (all $P<0.001)$. On the contrary, RANK expression was higher in triple negative tumors $(P<0.001)$. Using false discovery rate $<0.05,151$ and 1,207 genes were significantly correlated with tumor-expressed RANKL and RANK expression by immunohistochemistry, respectively. High RANKL expression within primary tumor was associated with pathways related to mammary gland development, bone resorption, T-cell proliferation and regulation of chemotaxis, while RANK expression was associated with immune response and proliferation pathways. At a median follow-up of 65 months, neither RANK nor RANKL expression within tumor was associated with disease free survival in pregnant or non-pregnant group.

Conclusions: Pregnancy increases RANKL expression both in normal breast and primary tumors. These results could guide further development of RANKL-targeted therapy.

\section{Introduction}

Receptor activator for nuclear factor $\mathrm{kB}$ ligand (RANKL) is a key factor in bone resorption. It binds to receptor activator for nuclear factor $\mathrm{\kappa B}$ (RANK) on the osteoclast to promote osteoclastogenesis, which results in bone destruction, osteoporosis and osseous metastasis [1]. Targeting the RANK/RANKL pathway emerged as a rational

\footnotetext{
* Correspondence: hatem.azim@bordet.be

${ }^{1}$ Breast Cancer Translational Research Laboratory (BCTL) J. C. Heuson, Institut Jules Bordet, Université Libre de Bruxelles, Boulevard de Waterloo 121,

Brussels, BE 1000, Belgium

2Department of Medicine, BrEAST Data Centre, Institut Jules Bordet,

Université Libre de Bruxelles (ULB), Brussels 1, Belgium

Full list of author information is available at the end of the article
}

strategy to arrest this process, and the anti-RANKL monoclonal antibody denosumab is currently approved in managing osteoporosis and preventing skeletal-related events secondary to bone metastases $[2,3]$.

Moreover, RANKL and its receptor have been shown to play a pivotal role in mammary gland development and in the increase of mammary stem cell pool during pregnancy [4]. Preclinical studies showed that RANKL is a major paracrine effector of progesterone's mitogenic action in the mammary epithelium [5,6]. More recently, data in humans suggested that RANKL expression fluctuates with serum progesterone, both on normal and malignant breast tissue [7]. Increased mammary tumor 
formation was observed in transgenic mice with gain of function in RANK following pregnancy or in wildtype mice following treatment with progesterone, a process that was arrested using a RANKL inhibitor [6]. Furthermore, RANKL was shown to be vital in mediating distant metastasis in breast cancer mice models [6,8]. Together, this evidence points to a fundamental role of RANK/ RANKL signaling in breast carcinogenesis.

Breast cancer arising at a young age is known to be biologically distinct, yet little progress has been made in identifying potential treatment targets [9]. Previous analysis by our group has suggested an association between breast cancer arising at young age and high RANKL mRNA expression [10]. On the other hand, young women are at a higher risk of breast cancer shortly after pregnancy and pregnancy-associated breast cancer is known to have poor prognosis $[11,12]$. While preclinical data have suggested a potential role of RANKL in mediating cancer initiation and progression associated with pregnancy [4], supporting clinical data in pregnant cancer patients are lacking.

In the current study, we evaluated for the first time the expression of RANK and RANKL using immunohistochemistry in young and pregnant breast cancer patients. Based on preclinical observations, we hypothesized that pregnancy would increase RANKL expression. We also evaluated gene expression patterns and activated pathways associated with RANK and RANKL expression.

\section{Methods}

\section{Study population}

A total of 195 patients with primary breast cancer were included in this analysis, of whom 65 were diagnosed during pregnancy. All patients were diagnosed and managed at the European Institute of Oncology (IEO) in Milan from 1996 to 2010. Information on the patient's characteristics and outcome was published previously [13]. Briefly, each pregnant patient was matched to two nonpregnant breast cancer patient controls according to age, tumor size, nodal status, date of diagnosis and whether neoadjuvant therapy was administered. All patients provided their consent to use their tissue samples for research purposes as per the IEO institutional policies. The study of biological features including genomic analysis was approved by the Ethics Committee of Institut Jules Bordet (Number 1782).

\section{Immunohistochemical staining}

Formalin-fixed tissues of primary breast surgeries were used for RANK and RANKL evaluation. For each patient, a hematoxylin and eosin-stained slide along with representative slides of the primary tumor and adjacent normal tissue ( $>1 \mathrm{~cm}$ from tumor) were shipped to Amgen Laboratories (Seattle, WA, USA) for immunohistochemical staining of RANK (N-1H8) and RANKL (M366) as described previously $[14,15]$, blinded to clinical information.
For each epitope, the staining score for tumor cells and adjacent normal epithelial cells was recorded separately. The percentage of immunostaining and the staining intensity ( 0 , negative; $1+$, weak; $2+$, moderate; and $3+$, strong) were recorded. An $\mathrm{H}$-score was calculated using the following formula:

$$
\begin{aligned}
\mathrm{H}-\text { score }= & (\% \text { of cells of weak intensity } \times 1) \\
& +(\text { percentage of cells of moderate intensity } \times 2) \\
& +(\text { percentage of cells of strong intensity } \times 3)
\end{aligned}
$$

The maximum $\mathrm{H}$-score would be 300 , corresponding to $100 \%$ of cells with strong intensity.

Evaluation of the histological subtype, histological grade and conventional breast cancer markers including estrogen receptor (ER), progesterone receptor (PgR), human epidermal growth factor receptor 2 (HER2) and Ki67 were performed at IEO. Breast cancer subtypes were defined using immunohistochemical surrogates as follows: luminal A like - ER and/or PgR(+), HER2(-), Ki67 <20\%; luminal B like - ER and/or PgR(+), HER2(-), Ki67 $\geq 20$; triple negative - ER, PgR and HER2(-), irrespective of Ki67 score; and HER2 - HER2(+), irrespective of ER, PgR or Ki67.

\section{Gene expression profiling}

Gene expression profiling using Affymetrix on the primary tumors of $85 \%$ of the included patients was published previously [16] and is publically available on Gene Expression Omnibus [GEO:GSE53031] [17].

To evaluate gene expression differences according to RANK and RANKL expression, we performed a linear regression model - testing genes that are associated with RANK, RANKL H-score; all treated as continuous variables. To control for multiple testing, we used the false discovery rate approach [18]. Genes presenting false discovery rate $<0.05$ were considered significantly associated with RANK or RANKL expression. We used a gene-set enrichment analysis (GSEA) to evaluate pathways associated with RANK or RANKL expression using the hypergeometric probabilities as published previously [19].

\section{Statistical analyses}

We evaluated the difference in expression of RANK/ RANKL H-score tested as a continuous variable on tumor and adjacent normal tissue between pregnant and nonpregnant patients. We also evaluated correlations between RANK/RANKL expression and the variables tumor size, nodal status, ER, PgR, HER2, Ki67 and breast cancer subtype using the Pearson chi-squared test. To evaluate factors that were independently associated with RANK/ RANKL expression, a linear regression model was constructed including all of the aforementioned variables.

We evaluated the association between tumor RANK and RANKL expression as a continuous variable and disease- 
free survival in a Cox regression model, for pregnant, nonpregnant and all patients combined.

Analyses were performed using SAS version 9.2 (SAS Institute, Cary, NC, USA) and R version 2.12.2 [20]. All tests were two-sided.

\section{Results}

Table 1 summarizes the patients' characteristics. As reported previously [13], no differences in clinicopathological features or breast cancer subtypes were observed between pregnant and nonpregnant patients. The median age was 36 years (range: 26 to 48 years). Information on prior parity and menstrual status at tumor sampling was not available.

RANK and RANKL expression by immunohistochemistry and association with pregnancy and clinicopathological features

RANKL staining was performed on 194 primary tumors (99\%) and 176 adjacent normal tissues (90\%); the results were positively correlated $(r=0.38, P<0.001)$.
The mean RANKL expression on normal epithelial cells was higher compared with that on tumor cells (50.19 vs. $16.13, P<0.001$ ) (Figure 1a). Patients diagnosed during pregnancy had significantly higher RANKL expression both on tumor cells (32.53 vs. $8.06, P<0.001)$ and adjacent normal tissue (87.29 vs. $32.88, P<0.001)$ (Figure 1a). In total, $18.7 \%$ of pregnant patients and $5.3 \%$ of nonpregnant patients had $\geq 10 \%$ of cells showing strong RANKL staining (score $3+$ ) on primary tumor, while $55.3 \%$ and $29.1 \%$ of pregnant and nonpregnant patients had at least $10 \%$ of cells $3+$ on adjacent normal tissue (Figure 2a,b). Additional file 1 shows examples of different staining intensities of RANKL. Among pregnant patients, a slightly lower expression of RANKL in both tumor and adjacent normal epithelial cells was observed in patients diagnosed in the third trimester of pregnancy (Figure S2a,b in Additional file 2).

RANK staining was performed on all 195 primary tumors and 181 adjacent normal tissue samples (92.8\%) and both results were positively correlated $(r=0.27, P<0.001)$. The mean RANK $\mathrm{H}$-score was higher for normal

Table 1 Patient characteristics and association with RANK and RANKL expression by immunohistochemistry

\begin{tabular}{|c|c|c|c|c|}
\hline & Pregnant $(n=65)$ & Nonpregnant $(n=130)$ & 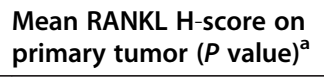 & $\begin{array}{l}\text { Mean RANK H-score on } \\
\text { primary tumor ( } P \text { value) }\end{array}$ \\
\hline \multicolumn{5}{|l|}{ Tumor size } \\
\hline$\leq 2 \mathrm{~cm}$ & $26(40 \%)$ & $52(40 \%)$ & 28.21 & 10.08 \\
\hline$>2 \mathrm{~cm}$ or $\mathrm{pT} \mathrm{x}^{\mathrm{b}}$ & $38(60 \%)$ & $78(60 \%)$ & $8.63(P=0.01)$ & $17.50(P=0.15)$ \\
\hline \multicolumn{5}{|l|}{ Nodal status } \\
\hline Negative & $28(43.1 \%)$ & $56(43.1 \%)$ & 24.51 & 14.12 \\
\hline Positive or $p N x^{b}$ & $37(56.9 \%)$ & $74(56.9 \%)$ & $9.04(P=0.03)$ & $14.36(P=0.96)$ \\
\hline \multicolumn{5}{|l|}{ Histological grade ${ }^{c}$} \\
\hline । & $4(6.2 \%)$ & $4(3.1 \%)$ & 70 & 7.5 \\
\hline$\|$ & $21(32.3 \%)$ & $43(33.1 \%)$ & 29.92 & 3.82 \\
\hline III & $36(55.3 \%)$ & $68(52.3 \%)$ & $6.25(P<0.001)$ & $22.2(P<0.001)$ \\
\hline \multicolumn{5}{|l|}{ ER } \\
\hline Positive & $43(66.1 \%)$ & $96(73.8 \%)$ & 20.43 & 5.43 \\
\hline Negative & $22(33.9 \%)$ & $34(26.2 \%)$ & $5(P=0.05)$ & $36.71(P<0.001)$ \\
\hline \multicolumn{5}{|l|}{$\mathrm{PgR}$} \\
\hline Positive & $42(64.6 \%)$ & $85(65.4 \%)$ & 24.11 & 5.09 \\
\hline Negative & $23(35.4 \%)$ & $45(34.6 \%)$ & $0.67(P<0.001)$ & $31.76(P<0.001)$ \\
\hline \multicolumn{5}{|l|}{ HER2 } \\
\hline Positive & $11(16.9 \%)$ & $23(17.7 \%)$ & 3.18 & 10.82 \\
\hline Negative & $54(83.1 \%)$ & $107(82.3 \%)$ & $18.79(P=0.1)$ & $14.95(P=0.53)$ \\
\hline \multicolumn{5}{|l|}{ PIK3CA mutation ${ }^{d}$} \\
\hline Yes & $10(15.4 \%)$ & $27(20.7 \%)$ & 24.03 & 6.3 \\
\hline No & $52(84.7 \%)$ & $102(79.3 \%)$ & $12.47(P=0.18)$ & $15.63(P=0.13)$ \\
\hline
\end{tabular}

ER, estrogen receptor; HER2, human epidermal growth factor receptor 2; PgR, progesterone receptor; RANK, receptor activator for nuclear factor KB; RANKL, receptor activator for nuclear factor $\mathrm{KB}$ ligand. ${ }^{\mathrm{a}}$ Not assessable in one patient in the pregnant group. ${ }^{\mathrm{b}} \mathrm{A}$ total of six patients had pathological tumor size is not evaluable (pTx) or pathological nodal status is not evaluable (pNx) (two pregnant and four nonpregnant). ${ }^{c}$ Not assessable in 19 patients (four pregnant and 15 nonpregnant). ${ }^{\mathrm{d}} \mathrm{Not}$ assessable in four patients (one pregnant and three nonpregnant). pTx (pathological tumor size is not evaluable) or pNx (pathological nodal status is not evaluable). 

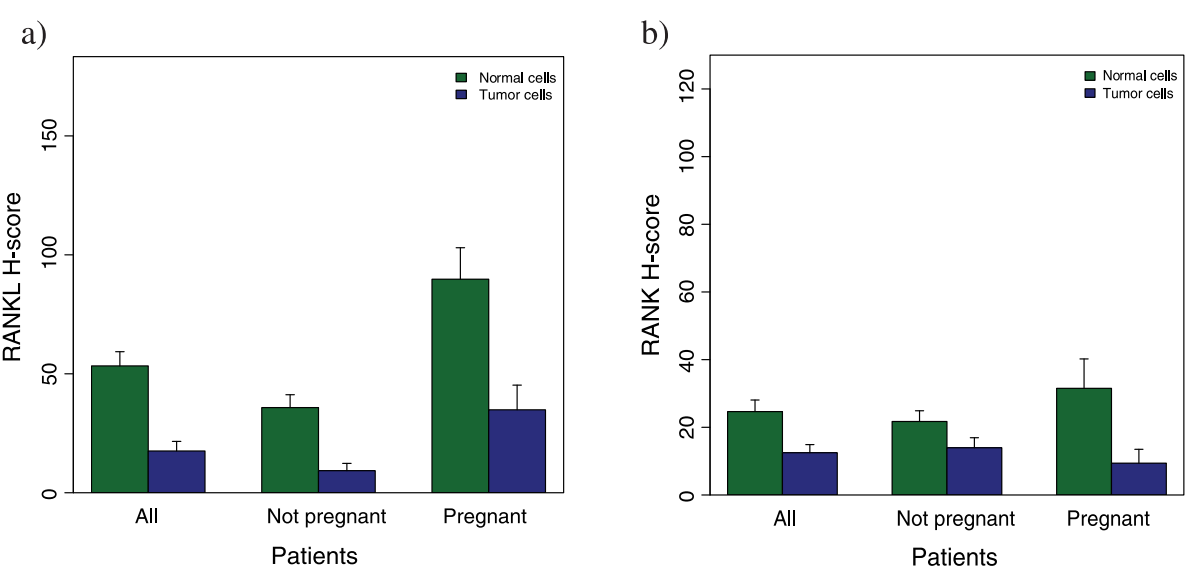

Figure 1 Differences in RANK and RANKL expression between pregnant and nonpregnant breast cancer patients. (a) Expression of RANKL by immunohistochemistry using the H-score ( $y$ axis) in all patients, pregnant patients and nonpregnant patients both in primary tumor (blue) and adjacent normal breast epithelial cells (green). Mean RANKL H-score was higher in normal breast epithelial cells compared with tumor cells; 50.19 versus $16.13, P<0.001$ in all patients; 32.88 versus $8.06, P<0.001$ in nonpregnant patients; and 87.29 versus 32.53 in pregnant patients, $P<0.001$. (b) Expression of RANK by immunohistochemistry using the $\mathrm{H}$-score (y axis) in all patients, pregnant patients and nonpregnant patients both in primary tumor (blue) and adjacent normal breast epithelial cells (green). Mean RANK H-score was higher in normal breast epithelial cells compared with tumor cells; 24.65 versus $12.48, P=0.003$ in all patients; 21.72 versus $13.95, P=0.07$ in nonpregnant patients; and 31.52 versus 9.41 in pregnant patients, $P=0.016$. RANK, receptor activator for nuclear factor $\mathrm{KB}$; RANKL, receptor activator for nuclear factor $\mathrm{KB}$ ligand.

a)

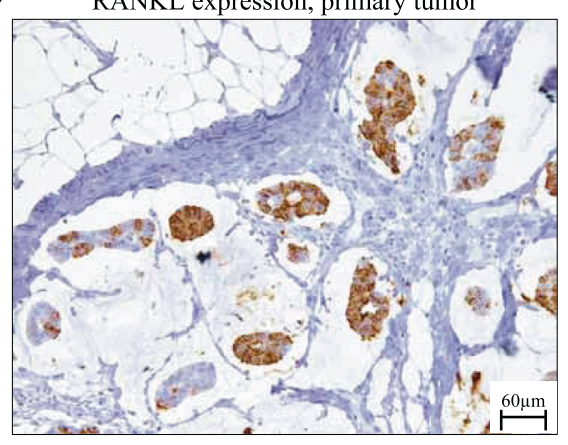

b)

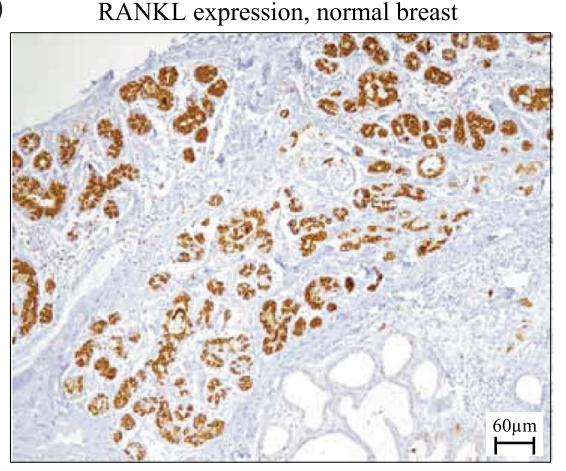

c)

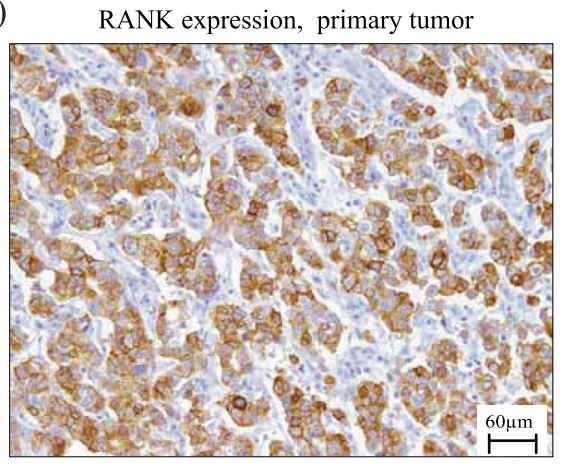

d)

RANK expression, normal breast

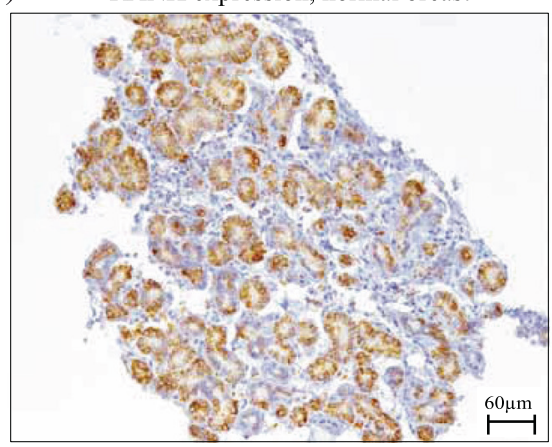

Figure 2 Examples of RANK and RANKL immunohistochemical staining on primary breast tumors and normal breast tissue. (a) RANKL expression on the primary tumor in a patient diagnosed with breast cancer during pregnancy (H-score: $270,80 \%$ of cells showing RANKL expression score 3+). (b) RANKL expression on adjacent normal epithelial cells in a patient diagnosed with breast cancer during pregnancy (H-score: 265, 80\% of cells showing RANKL expression score 3+). (c) RANK expression on the primary tumor in a young breast cancer patient not diagnosed during pregnancy (H-score: 140, $20 \%$ of cells showing RANK expression score 3+). (d) RANK expression on adjacent normal epithelial cells in a patient diagnosed with breast cancer during pregnancy (H-score: 210, 40\% of cells showing RANK expression score 3+). RANK, receptor activator for nuclear factor $\mathrm{KB}$; RANKL, receptor activator for nuclear factor $\mathrm{KB}$ ligand. 
versus tumor tissue (23.65 vs. $14.25, P=0.003$ ) (Figure $1 \mathrm{~b}$ ). No significant differences in RANK expression were observed between pregnant and nonpregnant patients; either on tumor tissue (13.74 vs. $14.51, P=0.88$ ) or adjacent normal epithelial cells (32.29 vs. $19.78, P=0.07$ ). Figure $2 \mathrm{c}, \mathrm{d}$ shows RANK expression on primary tumor and adjacent normal tissue. Additional file 3 shows other examples of different staining intensities of RANK. Among pregnant patients, a slightly lower RANK expression was observed in patients diagnosed in the first trimester of pregnancy (Figure S2c,d in Additional file 2).

Table 1 shows the association between RANK and RANKL expression on the primary tumor and clinicopathological features. For pregnant and nonpregnant patients, RANKL expression was higher in small $(P=0.01)$, well-differentiated $(P<0.001)$ and PgR-positive tumors $(P<0.001)$. On the contrary, higher RANK expression was observed in patients with poorly differentiated and hormone receptor-negative tumors (all $P<0.001$ ). RANKL expression was significantly higher in luminal A-like tumors (mean H-score: 45.45) compared with other subtypes, with the lowest expression observed in triple-negative subtypes (mean H-score: 0.23) (Figure 3a). Furthermore, the RANKL H-score was negatively correlated with Ki67 $(P<0.001)$ (Figure $3 \mathrm{~b})$. On the other hand, RANK expression was significantly higher in triple-negative tumors (mean H-score: 40.91) with the lowest expression observed in luminal A tumors (mean H-score: 4.68) (Figure 3c). A positive correlation was observed between RANK H-score and Ki67 (Figure 3d).

To evaluate factors independently associated with RANKL and RANK expression, an adjusted linear regression model showed that only pregnancy and high PgR expression were independently associated with high tumor RANKL expression (both $P<0.001$ ). On the other hand, breast cancer subtypes and administering neoadjuvant
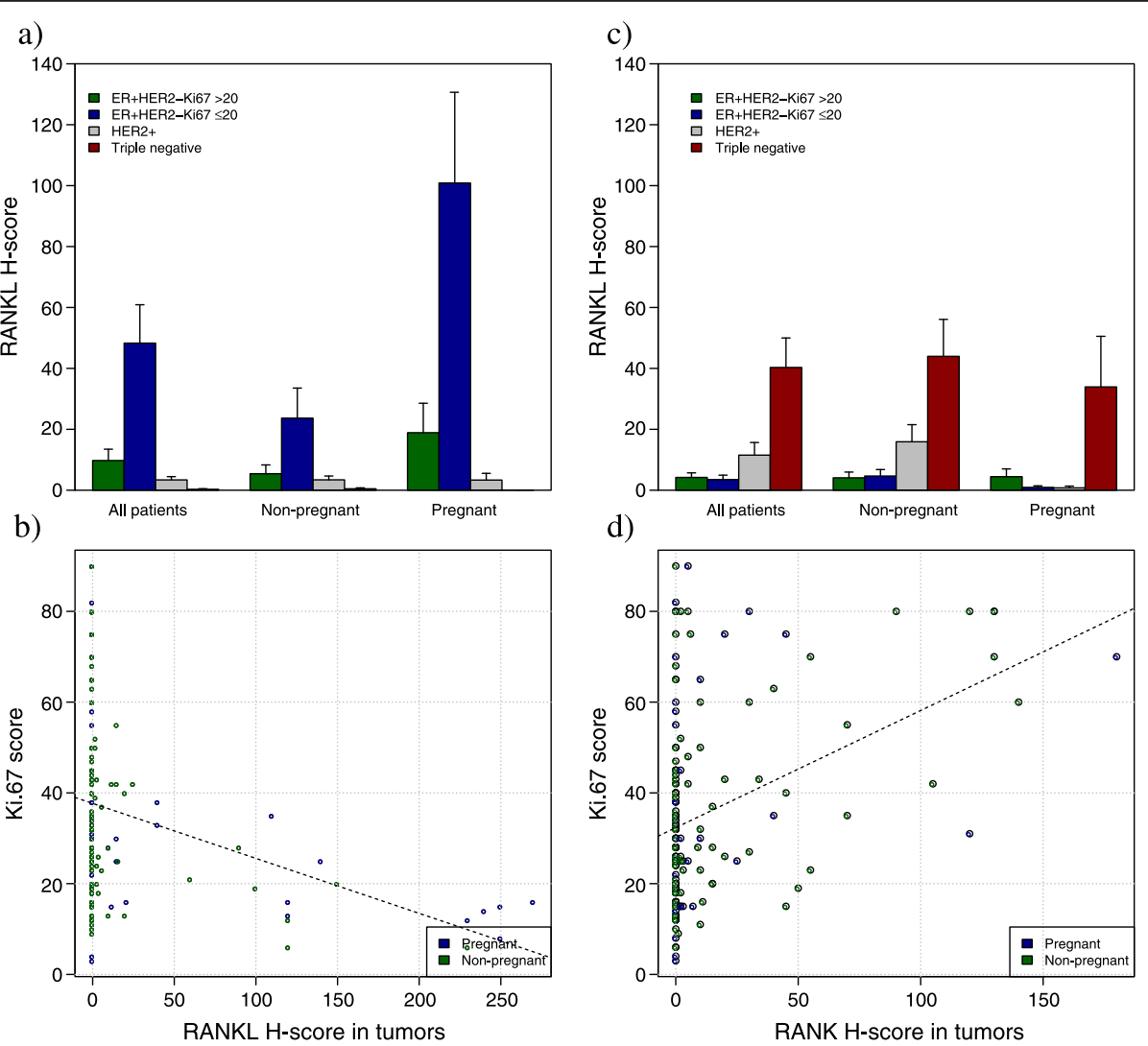

Figure 3 Expression of RANK and RANKL according to breast cancer subtype and correlation with Ki67. (a) Expression of RANKL by immunohistochemistry using the $\mathrm{H}$-score (y axis) according to breast cancer subtypes in all patients, nonpregnant patients and pregnant patients. RANKL expression was higher in luminal A tumors (ER+, HER2-, Ki67 $<20 \%$ ) compared with all other subtypes, particularly in pregnant patients $(P<0.0001)$, than in nonpregnant patients $(P=0.32)$. (b) Negative correlation between Ki67 score by immunohistochemistry (y axis) and RANKL $\mathrm{H}$-score ( $x$ axis) $(P<0.0001$, Pearson correlation $=-0.29$ ). (c) Expression of RANK by immunohistochemistry using the $\mathrm{H}$-score $(y$ axis) according to breast cancer subtypes in all patients, nonpregnant patients and pregnant patients. RANK expression was higher in triple-negative tumors compared with all other subtypes in all patients $(P<0.0001)$, nonpregnant patients $(P<0.0001)$ and pregnant patients $(P=0.05)$. (d) Positive correlation between Ki67 score by immunohistochemistry (y axis) and RANK H-score ( $x$ axis) $(P<0.0001$, Pearson correlation $=0.37)$. ER, estrogen receptor; HER2, human epidermal growth factor receptor 2; RANK, receptor activator for nuclear factor $\mathrm{KB}$; RANKL, receptor activator for nuclear factor $\mathrm{KB}$ ligand. 
chemotherapy were independently associated with RANK expression $(P<0.001)$ (Table 2$)$.

\section{Genes and pathways associated with RANK and RANKL} expression on primary breast tumors

Using false discovery rate $<0.05$, out of 18,665 evaluated genes 1,207 genes and 151 genes were significantly correlated (either positively or negatively) with RANK and RANKL H-score, respectively (Additional file 4). Almost perfect correlation was observed between tumor RANKL H-score and mRNA levels $(r=0.89, P<0.001)$ (Figure 4a). A weaker correlation, albeit significant, was observed between tumor RANK H-score and mRNA levels $(r=0.19$, $P=0.012$ ) (Figure 4b). GSEA showed that tumors expressing high levels of RANKL by immunohistochemistry had activated pathways; of particular relevance were those related to bone resorption, mammary gland development, regulation of chemotaxis and T-cell proliferation. On the other hand, high RANK expression was associated with several activated pathways, of relevance to immune response, and proliferation-related pathways (Additional file 5). These results were consistent even after adjusting the analysis for pregnancy status.

\section{Association between RANK and RANKL expression on the primary tumor and disease-free survival}

At a median follow-up of 65 months, $41.5 \%$ of the pregnant patients $(n=27)$ and $26.1 \%$ of the nonpregnant patients $(n=34)$ developed a disease-free survival event. Neither RANK nor RANKL were associated with the outcome when considering pregnant patients, nonpregnant patients or both groups combined (Additional file 6).

\section{Discussion}

This is the first study to evaluate the expression of RANK and RANKL in young and pregnant breast cancer patients. We found that pregnancy significantly increases RANKL expression both on the tumor and adjacent normal epithelial tissue. We also found that RANKL expression is associated with activation of important cancer-related pathways.

Recently, Pfitzer and colleagues reported on RANK/ RANKL expression in primary breast tumors analyzing 601 core biopsies collected from the neoadjuvant GeparTrio phase III trial, using the same antibodies and staining procedures as in the current analysis [14]. However, unlike our study where we did not use cutoff points to define RANK/RANKL positivity, Pfitzer and colleagues used an $\mathrm{H}$-score cutoff value $\geq 8.5$ to define patients with high expression. In their analysis, high RANK and RANKL expressions were observed in $14.5 \%$ and $6 \%$, respectively. In the current study, RANK and RANKL H-scores $\geq 8.5$ were observed in $28.5 \%$ and $12.3 \%$ respectively in the nonpregnant group and in $18.5 \%$ and $29.2 \%$ in the pregnant group. Previously, we reported higher RANKL mRNA expression in younger breast cancer patients [10]. The higher prevalence of RANK/RANKL expression in the current analysis could be due to the younger patient population in the current study (median age $=36$ years) compared with the GeparTrio trial (median age $=49$ years). It is important to note that in our analysis, RANK/RANKL expression was evaluated in surgical specimens as opposed to core biopsies (as in the GeparTrio trial). We have shown that both RANK and RANKL heterogeneously stain tumor and normal breast tissue (Additional files 1 and 3); therefore, it is also possible that the small core samples may underrepresent the incidence of RANK and RANKL expression.

We observed higher RANK expression in poorly differentiated and triple-negative tumors, with positive correlation between RANK H-score and Ki67 labeling index. Similar findings were also observed in the GeparTrio study and in earlier work using RANK mRNA expression $[14,21]$. This is consistent with preclinical work showing that RANK induces the expression of breast cancer stem and basal/stem cell markers [21]. On the other hand, we found higher expression of RANKL in the slowly proliferating luminal A-like tumors. Positive correlation was observed between RANKL H-score and

Table 2 Linear regression model showing clinicopathological factors that are independently associated with RANK and RANKL expression

\begin{tabular}{lll}
\hline & $\begin{array}{l}\text { Independent association } \\
\text { with increasing RANKL } \\
\text { expression (P value) }\end{array}$ & $\begin{array}{l}\text { Independent association } \\
\text { with increasing RANK } \\
\text { expression }(\boldsymbol{P} \text { value) }\end{array}$ \\
\hline Diagnosis during pregnancy (yes vs. no) & $<0.001$ \\
Tumor size ( $\leq 2 \mathrm{~cm}$ vs. $>2 \mathrm{~cm})$ & 0.23 \\
Nodal involvement (negative vs. positive) & 0.15 \\
Histological grade (I vs. II vs. III) & 0.09 \\
Breast cancer subtypes (luminal A vs. luminal B vs. HER2 vs. triple negative) & 0.75 \\
Increasing progesterone receptor expression by IHC (continuous variable) & $<0.001$ \\
Neoadjuvant chemotherapy (yes vs. no) & 0.19 \\
\hline
\end{tabular}



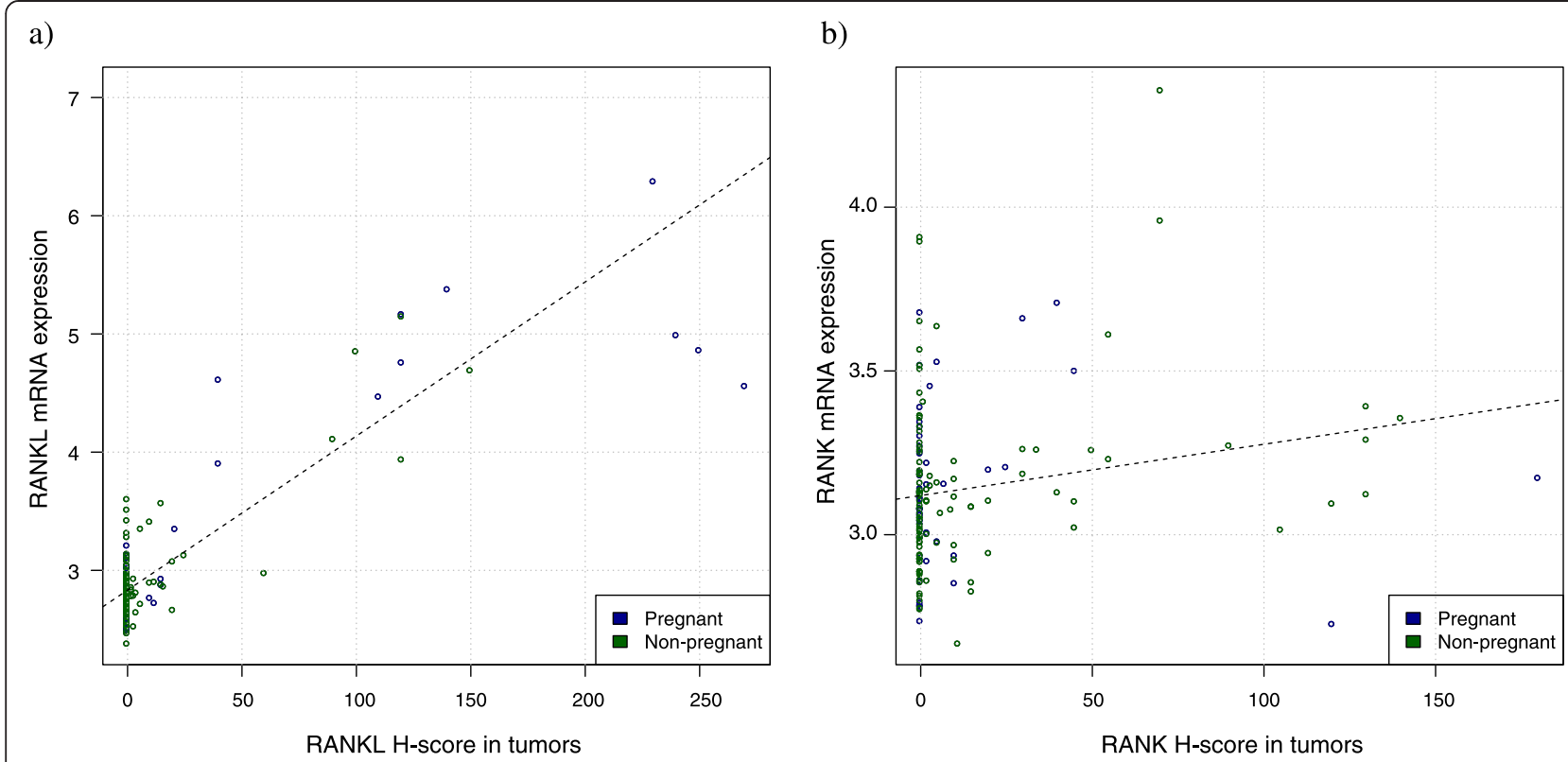

Figure 4 Correlation between RANK and RANKL H-score and mRNA levels. (a) Positive correlation between RANKL mRNA expression (y axis) and RANKL H-score by immunohistochemistry $(x$ axis) $(P<0.0001$, Pearson correlation $=0.89)$. (b) Positive correlation between RANK mRNA expression ( $y$ axis) and RANK H-score by immunohistochemistry ( $x$ axis) $(P=0.01$, Pearson correlation $=0.19)$. RANK, receptor activator for nuclear factor $K B$; RANKL, receptor activator for nuclear factor $K B$ ligand.

PgR scores (Figure S5a in Additional file 7), which is consistent with the preclinical data showing co-expression of RANKL and PgR on the normal breast, pre-invasive lesions and invasive lesions [6].

The striking difference in RANKL expression between pregnant and nonpregnant patients is intriguing. RANKL expression was significantly higher in both the normal epithelial and neoplastic cells arising during pregnancy. These results are consistent with preclinical data showing high expression of RANKL in normal breast tissue during pregnancy [4]. However, this is the first report to evaluate RANKL expression in tumors diagnosed during pregnancy in the clinical setting. We also found that RANK and RANKL expression differ according to the pregnancy trimester (Additional file 2), which was also observed in preclinical experiments [4].

The gene expression analysis provided confirmatory data regarding the known functions of RANK and RANKL and insights into the potential role(s) of RANK and RANKL in breast carcinogenesis. We observed a positive correlation between immunohistochemistry and mRNA expression, confirming the robustness of the performed assays. We also determined significant correlation between the RANKL H-scores and genes known to be related to RANKL function such as $P g R$ and parathyroid hormone-related protein (Figure S5b in Additional file 7). There was significant correlation between genes that we recently found to be related to breast cancer during pregnancy, such as insulin growth factor 1 and
TC1 (Figure S5c,d in Additional file 7), a positive regulator of the Wnt/beta-catenin signaling pathway [16]. Consistent with the known functions of RANKL, we observed activation of bone resorption and mammary gland development pathways in tumors with high RANKL expression. We also observed high expression of pathways related to T-cell proliferation. RANKL is known to enhance T-cell response and increase dendritic cell survival via binding to RANK [22]. RANKL expression on the tumor was associated with downregulation of proliferation and cell cyclerelated pathways, which is consistent with the clinical correlations that we observed, in which high RANKL was mainly observed in the slowly proliferative tumors. On the other hand, RANK expression was associated with activation of immune response and proliferation, again consistent with the high expression of RANK in the poorly differentiated, triple-negative tumors in which immunerelated pathways are emerging as important targets in this tumor subtype [23].

A limitation of our study is the small sample size, which might have masked a potential prognostic role of RANK or RANKL. Recently, neither was found to be predictive of pathological complete response in a large neoadjuvant study [14]. However, irrespective of the prognostic value of RANK and RANKL, their role as cancer targets is established. Another limitation is the lack of information on the phase of menstrual cycle at the time of tissue sampling, as evolving data suggest RANKL expression to vary across the menstrual cycle 
$[7,24]$. However, we believe that the striking differences in RANKL expression between pregnant and nonpregnant patients are unlikely to be significantly impacted by the unavailability of these data.

Our results underscore the relevance of RANKL as a potential target in breast cancer arising in young women. To further validate this concept, we are conducting a preoperative window trial evaluating the impact of the anti-RANKL denosumab on the biology of tumors arising in young women (D-BEYOND; NCT01864798). We also found that tumors diagnosed during pregnancy are more likely to express RANKL, raising the question of whether it could serve as a valid treatment target in these patients. To date we lack evidence supporting the use of denosumab in patients with primary breast cancer, yet several studies are currently ongoing to address this question including the predictive value of RANKL expression [25]. Another important finding is the high expression of RANKL in the normal breast of pregnant patients. Preclinical data support the important role of RANKL expression in breast cancer initiation, and given the known short-term risk of developing breast cancer following pregnancy it is plausible that RANKL expression on the normal breast would identify patients at high risk of developing breast cancer following pregnancy. This hypothesis is rather speculative and requires further investigation, but could potentially open a new venue for identifying high-risk women who are candidates for chemoprevention strategies.

\section{Conclusions}

This is the first study to evaluate the expression of RANKL in young and pregnant breast cancer patients. Our results indicate that pregnancy significantly increases RANKL expression independent of PgR or other factors; both on the primary tumor and on normal breast tissue. High expression of RANKL is also associated with activation of important cancer-related pathways. Our findings confirm the preclinical evidence suggesting RANKL as a potential breast cancer treatment target; particularly in young women and pregnancy-associated tumors.

\section{Additional files}

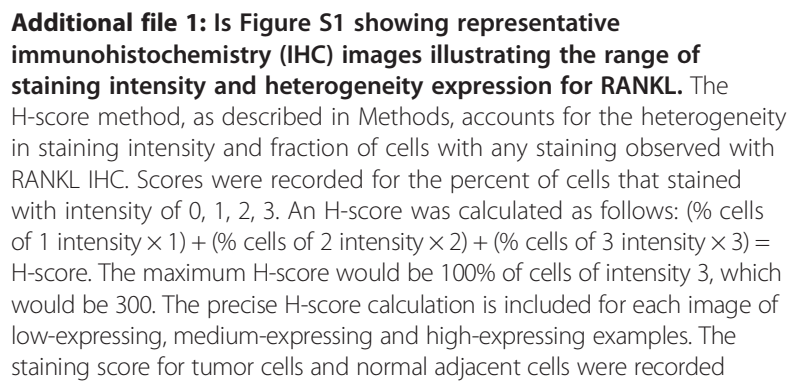

separately. Similar heterogeneity in RANKL staining intensity and fraction of positive cells was observed in both tumors and normal breast. (a) RANKL IHC of a breast tumor sample with relatively low expression ( $\mathrm{H}$-score $=21)$. (b). RANKL IHC of a breast tumor sample with medium expression $(\mathrm{H}$-score $=60)$. (c). RANKL IHC of a breast tumor sample with high expression $(\mathrm{H}$-score $=140)$. (b) and (c) represent the heterogeneous distribution of RANKL staining intensities within the same sample.

Additional file 2: Is Figure S2 showing (a) RANKL expression by immunohistochemistry on the primary tumor of pregnant patients according to trimester at breast cancer diagnosis. $y$ axis, mean $\mathrm{H}$-score and $95 \%$ confidence interval. Tumor diagnosed in the third trimester had the lowest RANKL expression (trimester $1+2 \mathrm{vs}$. trimester $3, P=0.04$ ). (b) RANKL expression by immunohistochemistry on adjacent normal epithelial cells of pregnant patients according to trimester at breast cancer diagnosis. $y$ axis, mean $\mathrm{H}$-score and $95 \%$ confidence interval. RANKL expression was lowest in the third trimester (trimester $1+2$ vs. trimester $3, P<0.001$ ). (c) RANK expression by immunohistochemistry on the primary tumor of pregnant patients according to trimester at breast cancer diagnosis. $y$ axis, mean $\mathrm{H}$-score and 95\% confidence interval. Tumor diagnosed in the first trimester had the lowest RANK expression (trimester 1 vs. trimester $2+3$, $P=0.4$ ). (d) RANK expression by immunohistochemistry on adjacent normal epithelial cells of pregnant patients according to trimester at breast cancer diagnosis. y axis, mean $\mathrm{H}$-score and $95 \%$ confidence interval. RANK expression was lowest in the first trimester (trimester 1 vs. trimester $2+3, P=0.27$ ).

Additional file 3: Is Figure $\mathrm{S3}$ showing representative IHC images illustrating the range of staining intensity and heterogeneity expression for RANK. IHC scoring was performed using the H-score method, which accounts for the heterogeneity in staining intensity and fraction of cells with any staining observed with both RANK and RANKL IHC. Scores were recorded for the percent of cells that stained with intensity of 0 , 1, 2, 3. An H-score was calculated as follows: (\% cells of 1 intensity $\times 1)+(\%$ cells of 2 intensity $\times 2)+(\%$ cells of 3 intensity $\times 3)=\mathrm{H}$-score. The maximum $\mathrm{H}$-score would be $100 \%$ of cells of intensity 3 which would be 300 . The precise $\mathrm{H}$-score calculation is included for each image of low-expressing, medium-expressing and high-expressing examples. The staining score for tumor cells and normal adjacent cells was recorded separately. Similar heterogeneity in RANK staining intensity and fraction of positive cells was observed in both tumors and normal breast. (a) RANK IHC of a breast tumor sample with relatively low expression $(H-s c o r e=20)$. (b). RANK IHC of a breast tumor sample with high expression $(\mathrm{H}-\mathrm{score}=70)$. (c). RANK IHC of a breast tumor sample with very high expression $(H-s c o r e=140)$. This image represents RANK expression detection at three different staining intensities within the same sample.

Additional file 4: Is a table presenting genes associated with RANK and RANKL expression by immunohistochemistry using the $\mathrm{H}$-score as a continuous variable.

Additional file 5: Is Figure S4 showing gene-set enrichment analysis showing upregulated pathways associated with RANKL (a) and RANK (b) expression by immunohistochemistry using the $\mathrm{H}$-score as a continuous variable.

Additional file 6: Is a table presenting univariate Cox regression analysis evaluating the effect of RANKL and RANK expression on disease-free survival.

Additional file 7: Is Figure $\mathrm{S} 5$ showing (a) positive correlation between PgR mRNA expression ( $y$ axis) and RANKL $\mathrm{H}$-score by immunohistochemistry ( $x$ axis) $(P<0.0001$, Pearson correlation $=0.35)$.

(b) Positive correlation between parathyroid hormone-related hormone mRNA expression (y axis) and RANKL H-score by immunohistochemistry ( $x$ axis) $(P<0.0001$, Pearson correlation $=0.47)$. (c) Positive correlation between IGF1 mRNA expression (y axis) and RANKL H-score by immunohistochemistry ( $x$ axis) $(P<0.0001$, Pearson correlation $=0.34)$. (d) Positive correlation between TC1 mRNA expression (y axis) and RANKL H-score by immunohistochemistry $(x$ axis $)(P<0.0001$, Pearson correlation $=0.32)$.

\section{Abbreviations}

ER: estrogen receptor; HER2: human epidermal growth factor receptor 2; IEO: European Institute of Oncology; PgR: progesterone receptor; RANK: receptor activator for nuclear factor KB; RANKL: receptor activator for nuclear factor $\mathrm{KB}$ ligand. 


\section{Competing interests}

HAA Jr, SL, MP and CS conducted clinical research supported by Amgen. DB and $\mathrm{BD}$ are employees in Amgen and hold stocks and stock options at Amgen. The remaining authors declare that they have no competing interests.

\section{Authors' contributions}

HAA Jr was responsible for the study design, collected clinical data, performed RNA extraction for gene expression, participated in the gene expression and statistical analyses, and wrote the initial draft of the manuscript. FAP participated in the study design, collected clinical data, participated in interpretation of the data and critically reviewed the first draft of the manuscript. SB performed gene expression and statistical analyses, participated in interpretation of the data and critically reviewed the first draft of the manuscript. DB carried out the pathological evaluation of RANKVRANKL, participated in interpretation of the data and critically reviewed the first draft of the manuscript. SL participated in the study design and critically reviewed the first draft of the manuscript. GV participated in the preparation of tissue samples, pathological evaluation of classic pathological parameters and interpretation of the data and critically reviewed the first draft of the manuscript. MP participated in the study design and critically reviewed the first draft of the manuscript. WCD participated in overall analysis of the study results and critically reviewed the first draft of the manuscript. GP carried out the preparation of tissue samples and pathological evaluation of classic pathological parameters, participated in interpretation of the data and critically reviewed the first draft of the manuscript. CS participated in study design, gene expression analysis and interpretation of the data and critically reviewed the first draft of the manuscript. All authors read and approved the final manuscript

\section{Authors' information}

GP and CS are co-last authors.

\section{Acknowledgements}

The authors would like to acknowledge the kind support of Fondazione Instituto Europea di Oncologia, in memory of Count Marco Marcello de Majno. Editing support was provided by Albert Y Rhee (Amgen) and Cactus Communications.

Amgen performed RANK and RANKL testing by immunohistochemistry and funded the transport of tissue samples from IEO to their laboratories. No other funding was provided. Amgen provided input in the interpretation of the data, and review of the manuscript.

\section{Author details}

'Breast Cancer Translational Research Laboratory (BCTL) J. C. Heuson, Institut Jules Bordet, Université Libre de Bruxelles, Boulevard de Waterloo 121, Brussels, BE 1000, Belgium. ²Department of Medicine, BrEAST Data Centre, Institut Jules Bordet, Université Libre de Bruxelles (ULB), Brussels 1, Belgium. ${ }^{3}$ Fertility and Procreation Unit, Department of Gynecologic Oncology, European Institute of Oncology, via Ripamonti 435, Milan 20141, Italy. ${ }^{4}$ Department of Pathology, Amgen Inc., 1201 Amgen Ct. West, Seattle, WA 98119, USA. ${ }^{5}$ Translational Breast Can cer Genomic Lab, Cancer Therapeutics Program, Peter MacCallum Cancer Centre, 2 St Andrews Place, East Melbourne, VIC 3002, Australia. ${ }^{6}$ Department of Pathology, European Institute of Oncology and University of Milan, via Ripamonti 435, Milan 20141, Italy. ${ }^{7}$ Department of Medical Oncology, Institut Jules Bordet, Université Libre de Bruxelles (ULB), Brussels 1, Belgium. ${ }^{8}$ Therapeutic Innovation Unit, Amgen Inc, 1201 Amgen Ct. West, Seattle, WA 98119, USA.

\section{Received: 23 September 2014 Accepted: 13 February 2015} Published online: 21 February 2015

\section{References}

1. Dougall WC. Molecular pathways: osteoclast-dependent and osteoclastindependent roles of the RANKL/RANK/OPG pathway in tumorigenesis and metastasis. Clin Cancer Res. 2012;18:326-35.

2. Stopeck AT, Lipton A, Body JJ, Steger GG, Tonkin K, de Boer RH, et al. Denosumab compared with zoledronic acid for the treatment of bone metastases in patients with advanced breast cancer: a randomized, doubleblind study. J Clin Oncol. 2010;28:5132-9.

3. Cummings SR, San Martin J, McClung MR, Siris ES, Eastell R, Reid IR, et al. Denosumab for prevention of fractures in postmenopausal women with osteoporosis. N Engl J Med. 2009;361:756-65.
4. Asselin-Labat ML, Vaillant F, Sheridan JM, Pal B, Wu D, Simpson ER, et al. Control of mammary stem cell function by steroid hormone signalling. Nature. 2010;465:798-802.

5. Schramek D, Leibbrandt A, Sigl V, Kenner L, Pospisilik JA, Lee HJ, et al. Osteoclast differentiation factor RANKL controls development of progestindriven mammary cancer. Nature. 2011;468:98-102.

6. Gonzalez-Suarez E, Jacob AP, Jones J, Miller R, Roudier-Meyer MP, Erwert R, et al. RANK ligand mediates progestin-induced mammary epithelial proliferation and carcinogenesis. Nature. 2010;468:103-7.

7. Hu H, Wang J, Gupta A, Shidfar A, Branstetter D, Lee O, et al. RANKL expression in normal and malignant breast tissue responds to progesterone and is up-regulated during the luteal phase. Breast Cancer Res Treat. 2014;146:515-23.

8. Tan W, Zhang W, Strasner A, Grivennikov S, Cheng JQ, Hoffman RM, et al. Tumour-infiltrating regulatory $T$ cells stimulate mammary cancer metastasis through RANKL-RANK signalling. Nature. 2011;470:548-53.

9. Azim HA Jr, Partridge AH. Biology of breast cancer in young women. Breast Cancer Res. 2014;16:427.

10. Azim HA Jr, Michiels S, Bedard PL, Singhal SK, Criscitiello C, Ignatiadis M, et al. Elucidating prognosis and biology of breast cancer arising in young women using gene expression profiling. Clin Cancer Res. 2012;18:1341-51.

11. Albrektsen G, Heuch I, Hansen S, Kvale G. Breast cancer risk by age at birth, time since birth and time intervals between births: exploring interaction effects. Br J Cancer. 2005;92:167-75.

12. Azim HA Jr, Santoro L, Russell-Edu W, Pentheroudakis G, Pavlidis N, Peccator FA. Prognosis of pregnancy-associated breast cancer: a meta-analysis of 30 studies. Cancer Treat Rev. 2012;38:834-42.

13. Azim HA Jr, Botteri E, Renne G, Dell'orto P, Rotmensz N, Gentilini O, et al. The biological features and prognosis of breast cancer diagnosed during pregnancy: a case-control study. Acta Oncol. 2012;51:653-61.

14. Pfitzner BM, Branstetter D, Loibl S, Denkert C, Lederer B, Schmitt WD, et al. RANK expression as a prognostic and predictive marker in breast cancer. Breast Cancer Res Treat. 2014;145:307-15.

15. Wood CE, Branstetter D, Jacob AP, Cline JM, Register TC, Rohrbach K, et al. Progestin effects on cell proliferation pathways in the postmenopausal mammary gland. Breast Cancer Res. 2013;15:R62.

16. Azim HA Jr, Brohee S, Peccatori FA, Desmedt C, Loi S, Lambrechts D, et al. Biology of breast cancer during pregnancy using genomic profiling. Endocr Relat Cancer. 2014;21:545-54.

17. http://www.ncbi.n/m.nih.gov/geo/.

18. Benjamini $Y$, Hochberg Y. Controlling the false discovery rate: a practical and powerful approach to multiple testing. J R Stat Soc B. 1995;57:289-300.

19. Brohee S, Faust K, Lima-Mendez G, Vanderstocken G, van Helden J. Network Analysis Tools: from biological networks to clusters and pathways. Nat Protocol. 2008;3:1616-29.

20. www.r-project.org.

21. Palafox M, Ferrer I, Pellegrini P, Vila S, Hernandez-Ortega S, Urruticoechea A, et al. RANK induces epithelial-mesenchymal transition and stemness in human mammary epithelial cells and promotes tumorigenesis and metastasis. Cancer Res. 2012;72:2879-88.

22. Cheng ML, Fong L. Effects of RANKL-targeted therapy in immunity and cancer. Front Oncol. 2014;3:329.

23. Andre F, Dieci MV, Dubsky P, Sotiriou C, Curigliano G, Denkert C, et al. Molecular pathways: involvement of immune pathways in the therapeutic response and outcome in breast cancer. Clin Cancer Res. 2013;19:28-33.

24. Haynes BP, Viale G, Galimberti V, Rotmensz N, Gibelli B, Smith IE, et al. Differences in expression of proliferation-associated genes and RANKL across the menstrual cycle in estrogen receptor-positive primary breast cancer. Breast Canc Res Treat. 2014;148:327-35.

25. Azim H, Azim HA Jr. Targeting RANKL in breast cancer: bone metastasis and beyond. Expet Rev Anticancer Ther. 2013;13:195-201. 